\title{
Pulmonary pathology of severe acute respiratory syndrome in Toronto
}

\author{
David M Hwang ${ }^{1,2,3}$, Dean W Chamberlain ${ }^{1,2,3}$, Susan M Poutanen ${ }^{1,3,4}$, Donald E Low ${ }^{1,3,4,5}$, \\ Sylvia L Asa ${ }^{1,2,3}$ and Jagdish Butany ${ }^{1,2,3}$ \\ ${ }^{1}$ Toronto Medical Laboratories, Toronto, Ontario, Canada; ${ }^{2}$ Department of Pathology, University Health \\ Network, Toronto, Ontario, Canada; ${ }^{3}$ Department of Laboratory Medicine and Pathobiology, Toronto, Ontario, \\ Canada; ${ }^{4}$ Department of Microbiology, Mount Sinai Hospital, Toronto, Ontario, Canada and ${ }^{5}$ Department of \\ Medicine, University of Toronto, Toronto, Ontario, Canada
}

\begin{abstract}
The severe acute respiratory syndrome (SARS) pandemic in Toronto resulted in a large number of autopsies on its victims. We describe the pulmonary pathology of patients who died in the 2003 Toronto outbreak. Autopsy material from the lungs of 20 patients who died between March and July 2003 were characterized by histology, molecular biology, and immunohistochemistry for cytokeratins, thyroid transcription factor-1, CD68, EpsteinBarr virus, cytomegalovirus, and human herpes simplex viruses. Matched controls were obtained from patients who died of other causes over the same interval. The mean duration of illness was 27 days (range 5-108 days). Post-mortem lung tissues from 19 of 20 patients with probable SARS were positive for SARS-associated coronavirus by RT-PCR. Histologically, all patients showed varying degrees of exudative and proliferative phase acute lung injury, evidenced in conventional and immunohistochemical stains by edema, inflammatory infiltrate, pneumocyte hyperplasia, fibrinous exudates, and organization. Eight of 20 patients showed predominantly a diffuse alveolar damage pattern of acute lung injury, six showed predominantly an acute fibrinous and organizing pneumonia pattern, and the remainder showed an admixture of the two patterns. Squamous metaplasia and scattered multinucleate giant cells were present in most cases. Vascular fibrin thrombi were a common finding and were often associated with pulmonary infarcts. Special stains demonstrated vascular endothelial damage of both small- and mid-sized pulmonary vessels. Two cases were complicated by invasive fungal disease consistent with Aspergillosis, and another by coinfection with cytomegalovirus. Our findings indicate that the lungs of patients who die of SARS are almost always positive for the SARS-associated coronavirus by RT-PCR, and may show features of both diffuse alveolar damage and acute fibrinous and organizing pneumonia patterns of acute injury. Cases of SARS may be complicated by coexistent infections and therapy-related lung injury.
\end{abstract}

Modern Pathology (2005) 18, 1-10, advance online publication, 23 July 2004; doi:10.1038/modpathol.3800247

Keywords: severe acute respiratory syndrome; diffuse alveolar damage; acute respiratory distress syndrome; lung pathology

The outbreak of severe acute respiratory syndrome (SARS) in Toronto resulted in 44 deaths among 375 probable and suspect cases between March and July $2003^{1-3}$ (http://www.hc-sc.gc.ca/pphb-dgspsp/sarssras/cn-cc/20030903_e.html). Caused by a newly discovered SARS-associated coronavirus (SARS$\mathrm{CoV}),{ }^{4-7}$ SARS is characterized largely by constitutional symptoms (fever, chills, myalgia) and lower respiratory tract symptoms, from cough and dys-

Correspondence: Dr J Butany, MBBS, MS, FRCPC, Department of Pathology, EC4-301, 200 Elizabeth Street, Toronto, Ontario, Canada M5G 2C4.

E-mail: jagdish.butany@uhn.on.ca

Portions of this work were presented at the United States and Canadian Academy of Pathology, Vancouver, BC, 2004.

Received 2 April 2004; revised 29 June 2004; accepted 30 June 2004; published online 23 July 2004 pnea, to rapidly progressive respiratory failure requiring intubation and mechanical ventilation..$^{1,2,8,9}$ Initial reports of the pulmonary pathology of SARS have described histologic findings in limited numbers of patients up to 3 weeks after the onset of symptoms. ${ }^{10-15}$ We describe here the pulmonary pathology of SARS in 20 patients who died in the Toronto outbreak, up to 108 days after the onset of symptoms.

\section{Materials and methods}

\section{Patients}

Post-mortem examinations were performed between March and July 2003 on 20 patients who met the World Health Organization (WHO) and Health 
Canada case definitions for probable SARS. ${ }^{2}$ The patients died at several facilities within the Greater Toronto Area, but post-mortem examinations were conducted by staff pathologists at the Toronto General Hospital of the University Health Network in Toronto, Canada, with the majority conducted by one pathologist (JB). Early in the course of the outbreak, autopsies were restricted to in situ chest examination (three cases). As understanding of the mode of transmission of SARS progressed, complete examinations were performed with appropriate safety precautions (N95/N97 masks, disposable hoods, visors, gowns, aprons, and gloves) (17 cases). Post-mortem tissues were sampled for histology, microbiology, electron microscopy, and molecular biology. Lung tissues from 22 SARS-CoV-negative patients who presented with significant lower respiratory tract symptoms and signs, and who died during the same period were similarly examined for comparison.

\section{RT-PCR for SARS-CoV}

RT-PCR detection of the SARS-CoV was performed as described in Mazzulli et al. ${ }^{16}$ In brief, tissues were homogenized in lysis buffer and RNA isolated with the RNeasy Mini Kit (Qiagen, Mississauga, Canada), in a sample elution volume of $30 \mu \mathrm{l}$. RT-PCR was performed using the RealArt HPA-Coronavirus LightCycler RT Reagents Assay (Artus GmbH, Hamburg, Germany) on a LightCycler real-time platform (Roche Diagnostics, Laval, Canada), which detects an 80-bp amplicon of the SARS-CoV polymerase gene, using primers published by the BernhardNocht Institute (Hamburg, Germany) and posted at the World Health Organization Website (URL: http:// www.who.int/csr/sars/primers/en).

\section{Histology and Immunohistochemistry}

Hematoxylin and eosin-stained slides of formalinfixed, paraffin-embedded post-mortem lung tissue were reviewed (mean 13 blocks/case; range 2-21 blocks) for each patient by at least two pathologists (SLA, JB, DWC, DMH). Histologic features were scored for the percentage of tissue involved (1-1$24 \%, 2-25-49 \%, 3-50-74 \%, 4-75-100 \%$ ), as described by Franks et al, ${ }^{12}$ based on assessment of all histologic sections for each case. Representative sections were selected and stained with BrownBrenn Gram, Ziehl-Nielsen, Gomori methenamine silver stains for microorganisms, and with elastic trichrome and Martius scarlet blue stains. Immunohistochemical stains for herpes simplex virus (HSV) 1 and 2, cytomegalovirus (CMV), and Epstein-Barr virus latent membrane protein-1 (EBV LMP-1), cytokeratin (AE1/AE3), thyroid transcription factor-1 (TTF-1), and CD68 were performed on one representative block of paraffin-embedded tissue for each case, using the Ventana stainer (Ventana
Systems, Tuscon, AZ, USA), with heat or protease antigen retrieval as necessary.

\section{Results}

\section{Patient Characteristics}

Characteristics of the SARS-CoV positive and comparison groups are summarized in Table 1. A total of

Table 1 Patient characteristics

\begin{tabular}{|c|c|c|}
\hline & $\begin{array}{l}\text { SARS-CoV- } \\
\text { positive group }\end{array}$ & $\begin{array}{l}\text { Comparison } \\
\text { group }\end{array}$ \\
\hline \multicolumn{3}{|l|}{ Age (years) } \\
\hline Mean & 68.1 & 69.7 \\
\hline Range & $43-99$ & $46-97$ \\
\hline \multicolumn{3}{|l|}{$\begin{array}{l}\text { Gender distribution } \\
\text { Duration of illness (days) }\end{array}$} \\
\hline Mean & 26.8 & 8.0 \\
\hline Median & 23.5 & 6 \\
\hline Range & $5-108$ & $1-35$ \\
\hline $\begin{array}{l}\text { Cases of } 14 \text { days' duration } \\
\text { or less }\end{array}$ & $5 / 20(25.0 \%)$ & $19 / 22(86.4 \%)$ \\
\hline $\begin{array}{l}\text { Cases with histologic features } \\
\text { of diffuse alveolar damage }\end{array}$ & $20 / 20(100 \%)$ & $11 / 22(50 \%)$ \\
\hline
\end{tabular}

Table 2 Extent of lung injury by duration of illness in SARS-CoVpositive patients

\begin{tabular}{lccc}
\hline & \multicolumn{2}{c}{ Extent, mean score $\mathrm{a}^{\mathrm{a}}$} & \multirow{2}{*}{$\mathrm{P}^{\mathrm{b}}$} \\
\cline { 2 - 3 } & $\begin{array}{c}\leq 14 \text { days' } \\
\text { duration }\end{array}$ & $\begin{array}{c}>14 \text { days, } \\
\text { duration }\end{array}$ & \\
\hline Acute fibrinous exudates & 2.80 & 1.67 & $<0.01$ \\
$\begin{array}{l}\text { Airspace edema } \\
\text { Organizing exudates }\end{array}$ & 1.80 & 1.47 & $\mathrm{NS}$ \\
Pneumocyte hyperplasia & 0.80 & 2.60 & $<0.01$ \\
& 1.60 & 3.27 & $<0.01$ \\
\hline
\end{tabular}

${ }^{\mathrm{a}}$ Individual scores for each case were assigned as described in Materials and methods.

${ }^{\mathrm{b}} P$-value by two-tailed $t$-test; NS $=$ not significant.

Table 3 Summary of other histologic features in lungs of SARS patients

\begin{tabular}{|c|c|c|}
\hline & \multicolumn{2}{|c|}{ Percentage of cases with feature } \\
\hline & $\begin{array}{c}\leq 14 \text { days } \\
\text { duration }\end{array}$ & $\begin{array}{c}>14 \text { days } \\
\text { duration }\end{array}$ \\
\hline Squamous metaplasia & 40 & 87 \\
\hline Multinucleated cells & 20 & 73 \\
\hline Acute bronchopneumonia & 20 & 60 \\
\hline Hemorrhage & 80 & 87 \\
\hline Fibrin thromboemboli & 80 & 87 \\
\hline Pulmonary infarcts & 20 & 73 \\
\hline
\end{tabular}


a
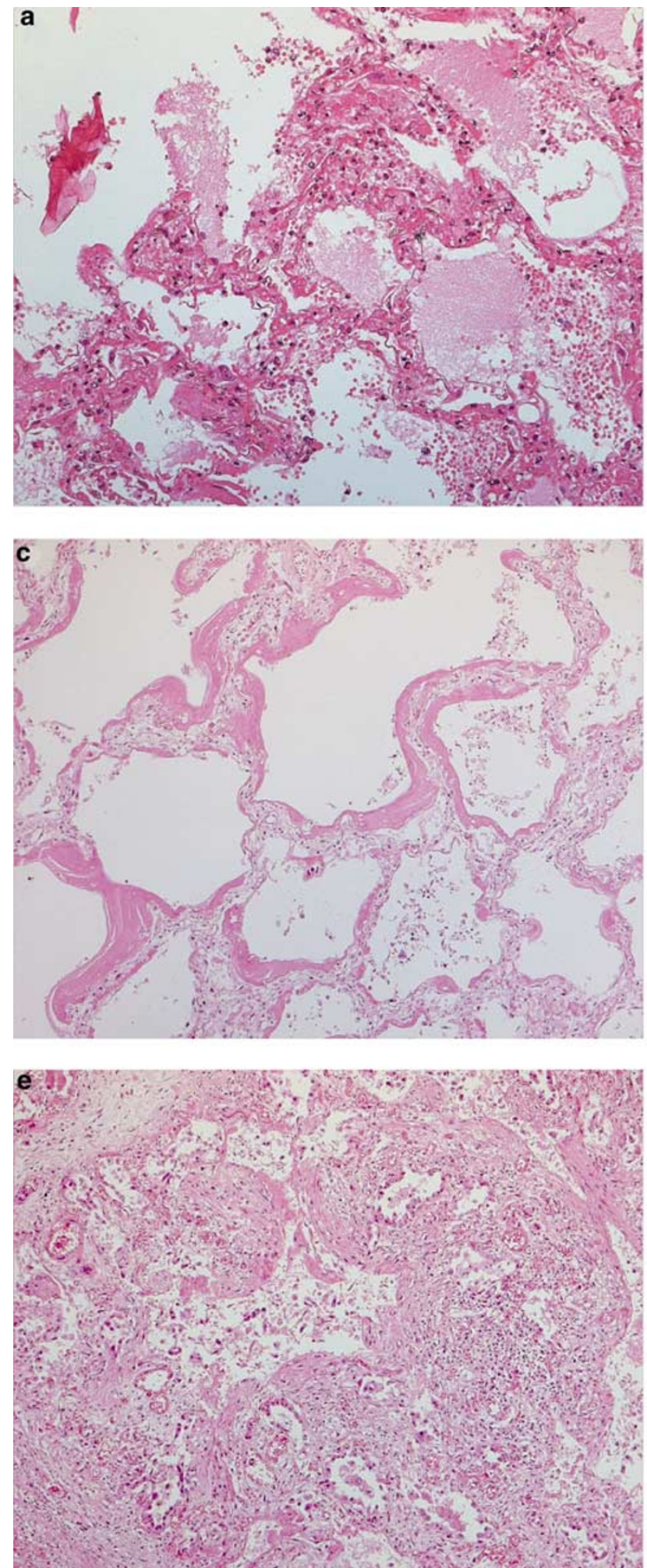
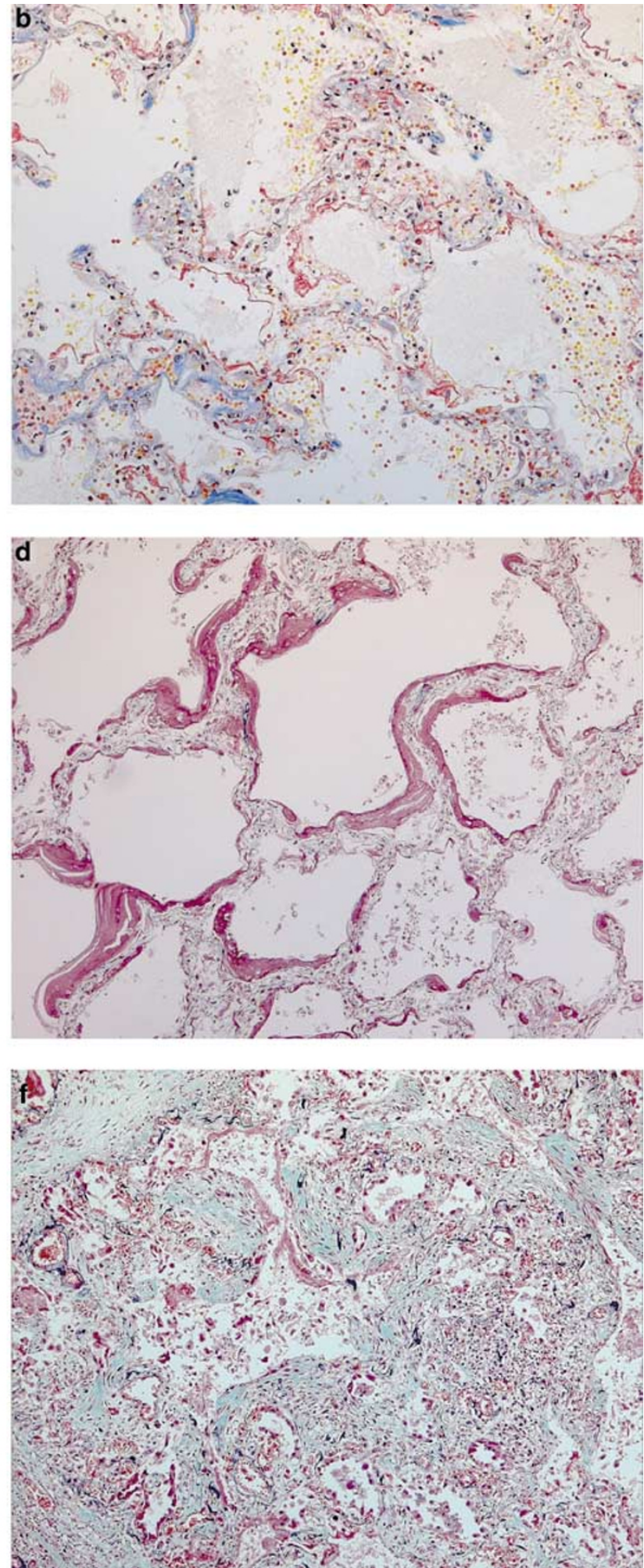

Figure 1 Diffuse alveolar damage pattern of lung injury in SARS patients. (a) Early exudative phase diffuse alveolar damage showing vascular congestion, with interstitial and airspace edema and inflammatory cell infiltrates (H\&E, original magnification $\times 200)$; (b) the same field showing fibrinous exudates by Martius scarlet blue stain (original magnification $\times 200$ ); (c, d) exudative phase diffuse alveolar damage, with hyaline membranes (c, H\&E, original magnification $\times 200$; d, elastic trichrome, original magnification $\times 200)$; $(\mathbf{e}, \mathbf{f})$ organizing phase diffuse alveolar damage $(\mathbf{e}, \mathrm{H} \& \mathrm{E}$, original magnification $\times 100$; $\mathbf{f}$, elastic trichrome, original magnification $\times 100)$. 

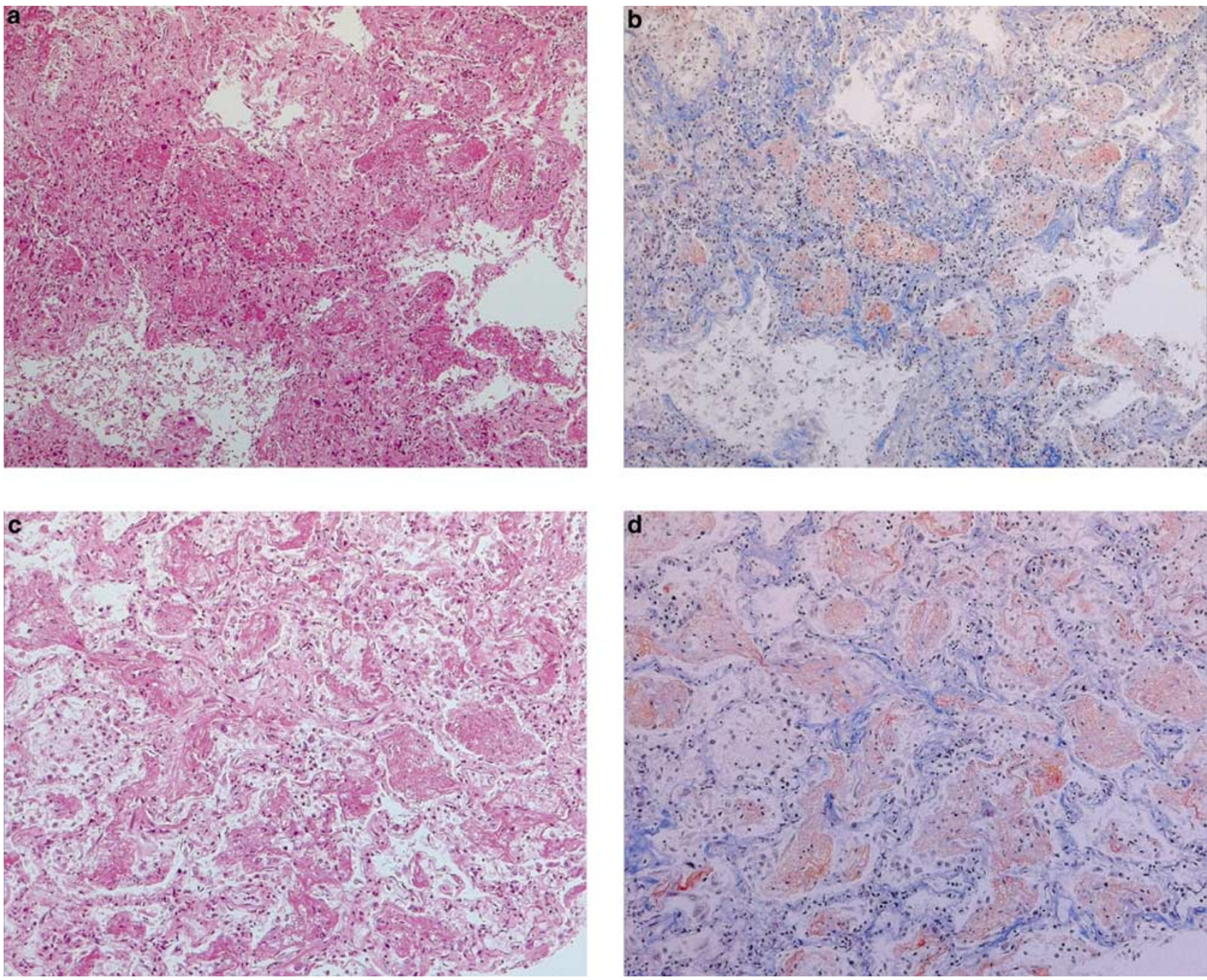

Figure 2 Acute fibrinous and organizing pneumonia pattern, acute exudative phase. Fibrinous exudates form 'balls' in alveolar spaces, rather than the hyaline membranes classically seen in diffuse alveolar damage pattern lung injury. (a, c) H\&E; (b, d) Martius scarlet blue; all frames at $\times 100$ original magnification.

11 women and nine men who died of probable SARS and who tested positive for SARS-CoV were included in this study. Of these, 19 tested positive for SARS-CoV in post-mortem lung tissue (duration of illness, 5-51 days), while one patient (duration of illness, 108 days) was only positive for the virus in ante-mortem clinical specimens, but not in postmortem tissues. Details concerning the RT-PCR results for eleven of these patients have been published elsewhere. ${ }^{16}$ The mean age was 68 years (range 43-99 years), and mean duration of illness from onset of symptoms to death was 27 days (range 5-108 days; median 23.5 days). The mean age of patients who died within 14 days of the onset of symptoms was 70 years, compared with 68 years for those with duration of illness in excess of 14 days.

None of the patients in the comparison group of 22 patients were positive for the SARS-CoV by RTPCR. Within this group, the mean age was 70 years (range 46-97 years), with an equal number of males and females. The mean duration of illness was somewhat shorter than the SARS-CoV-positive group, at 8 days, with only three patients surviving beyond 14 days.

\section{Histologic Characteristics of SARS}

The pulmonary histologic features of the 20 SARSCoV-positive cases are summarized in Tables 2 and 3. Eight patients showed predominantly a diffuse alveolar damage pattern of lung injury, with formation of hyaline membranes and interstitial thickening (Figure 1). Six cases showed a predominantly acute fibrinous and organizing pneumonia pattern of injury, as evidenced by formation of fibrin 'balls' within airspaces rather than hyaline membranes, and by an organizing pneumonia pattern of fibrosis ${ }^{17}$ (Figures 2 and 3). The remaining cases showed variable degrees of both patterns. As expected, cases 

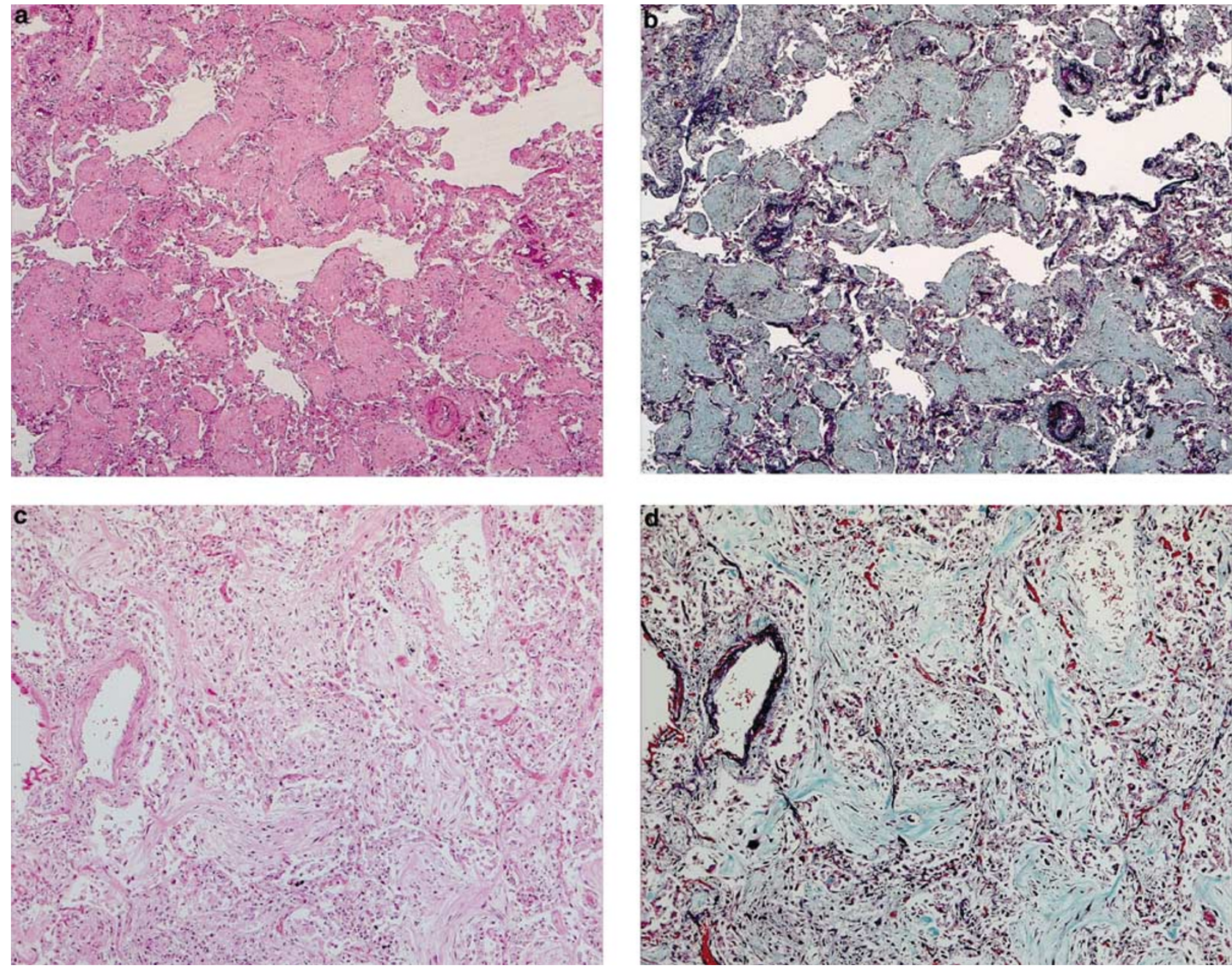

Figure 3 Acute fibrinous and organizing pneumonia pattern, organizing phase. Fibromyxoid plugs in bronchioles and alveolar spaces, with minimal alveolar septal thickening. (a) H\&E, original magnification $\times 40$; (b) elastic trichrome (same field as (a)), original magnification $\times 40$; (c) H\&E, original magnification $\times 100$; (d) elastic trichrome (same field as (c)), original magnification $\times 100$.

of shorter duration (14 days' duration or less) had more widespread acute injury (mean extent score 2.80 vs 1.67) than those of longer duration (more than 14 days), while those of longer duration showed more extensive organization (Table 2). All cases of 10 or less days' duration showed a predominantly diffuse alveolar damage pattern of injury, while all cases showing an acute fibrinous and organizing pneumonia-predominant pattern were of greater than 20 days' duration.

In contrast, only 11 of 22 patients (50\%) in the comparison group showed features of diffuse alveolar damage. Of these 11 patients, 10 showed a diffuse alveolar damage-predominant pattern, with only one patient showing admixed areas of acute fibrinous and organizing pneumonia (duration of illness $=16$ days). Among these 11 patients, eight $(73 \%)$ died within 14 days of onset of illness, compared with only five patients $(25 \%)$ in the SARS group. Of the eight patients in the control group who died within 14 days, at least five showed evidence suggesting underlying bacterial sepsis (three cases severe acute bronchopneumonia, one case acute pyelonephritis, one case pseudomembranous colitis), while two showed evidence of myocardial infarction. The extent of acute diffuse alveolar damage was similar between groups (mean score 1.95 in SARS group vs 1.55 in the comparison group; $P=0.25$ ), although among patients with duration of illness $\leq 14$ days, there was more widespread acute diffuse alveolar damage in SARS patients than in the comparison group (mean score 2.80 vs 1.38; $P=0.001)$. Consistent with the shorter mean duration of illness among the comparison group, SARS patients as a whole also showed a greater frequency of organizing lung injury than the comparison group (95\% of patients vs $36 \%$ ), and other histologic findings typically associated with organizing phase diffuse alveolar damage were also more prominent in the SARS group as a whole than 
6

in the comparison group, including pneumocyte hyperplasia (mean score 2.85 vs 1.27 , respectively), squamous metaplasia (present in $75 \%$ of SARS cases, vs $9 \%$ of control cases), and multinucleated giant cells (60 vs 36\%).

Within the SARS group, pneumocyte hyperplasia, with or without associated cytologic atypia, was more prominent in SARS cases of longer duration (Table 2), as were squamous metaplasia and multinucleate giant cells (Table 3 and Figure 4). Characterization of the multinucleate giant cells by immunohistochemistry found the cells to be positive for CD68, but cytokeratin or TTF-1-positive multinucleated cells were not identified. Foci of bronchopneumonia were somewhat more frequent in cases of longer duration, but where present, were relatively minimal in their extent. Gram and ZiehlNielsen stains failed to identify causative bacterial organisms in these cases. Gomori methenamine silver stain revealed invasive fungal disease consistent with Aspergillosis in two cases (see below). Immunohistochemical stains for HSV1 \& 2, EBV and CMV were negative in all cases except one (see below).

Vascular injury was a prominent feature in many SARS cases, but was also observed in the majority of comparison group cases exhibiting diffuse alveolar damage. While not always readily apparent on H\&E sections, staining with Martius scarlet blue demonstrated endothelial injury/denudation of small vessels, with intravascular fibrin deposition, and edema and extravasation of red blood cells in areas of acute diffuse alveolar damage (Figure 5). Fibrin thrombi in various stages of organization were also present in 17 of 20 SARS cases, while pulmonary infarcts were identified in 12 cases (Figure 6). Two cases showed extensive infarcts involving more than $25 \%$ of the tissue sampled.

\section{Pulmonary Coinfections in SARS Patients}

Three SARS patients showed definitive evidence of coinfection with other microorganisms. All three of these patients had been given high-dose steroids as part of the treatment for SARS. Of these, two patients, both 78-year-old female patients, showed bilateral coinfection with fungal organisms showing septate hyphae and acute angle branching, consistent with Aspergillus spp. (Figure 7a). Angioinvasion was more prominent in one case (Figure $7 \mathrm{~b}$ ) and was associated with extensive infarction of the lung. The third patient showed patchy acute pneumonia with large cells containing abundant amphophilic cytoplasm and prominent red-purple intranuclear inclusions surrounded by a clear halo (Figure 7c). These were confirmed to be CMV inclusions by immunohistochemistry (Figure $7 \mathrm{~d}$ ). Immunohistochemical stains for other viruses (HSV1, HSV2, EBV) were negative.
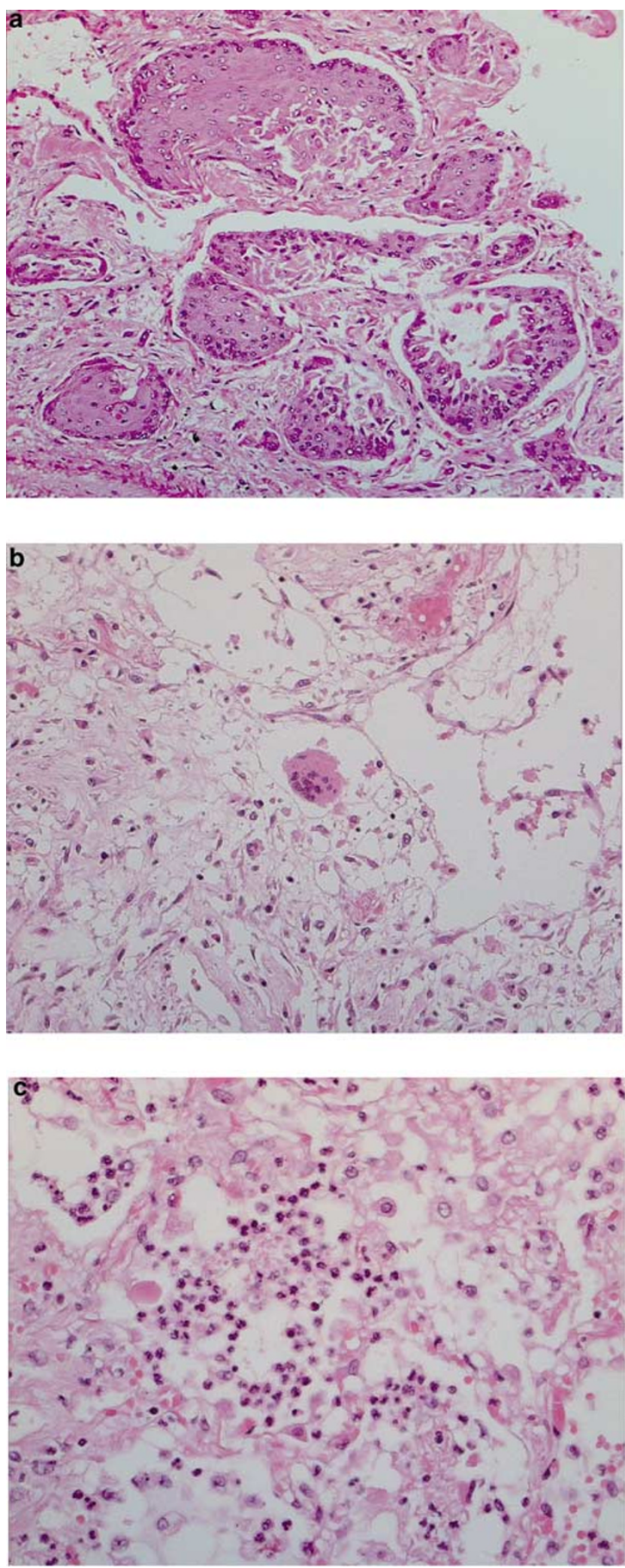

Figure 4 Other histologic features of SARS in the lung. (a) Squamous metaplasia (H\&E, original magnification $\times 200$ ); (b) multinucleate giant cell (H\&E, original magnification $\times 200)$; (c) focal acute bronchopneumonia (H\&E, original magnification $\times 400$ ) 

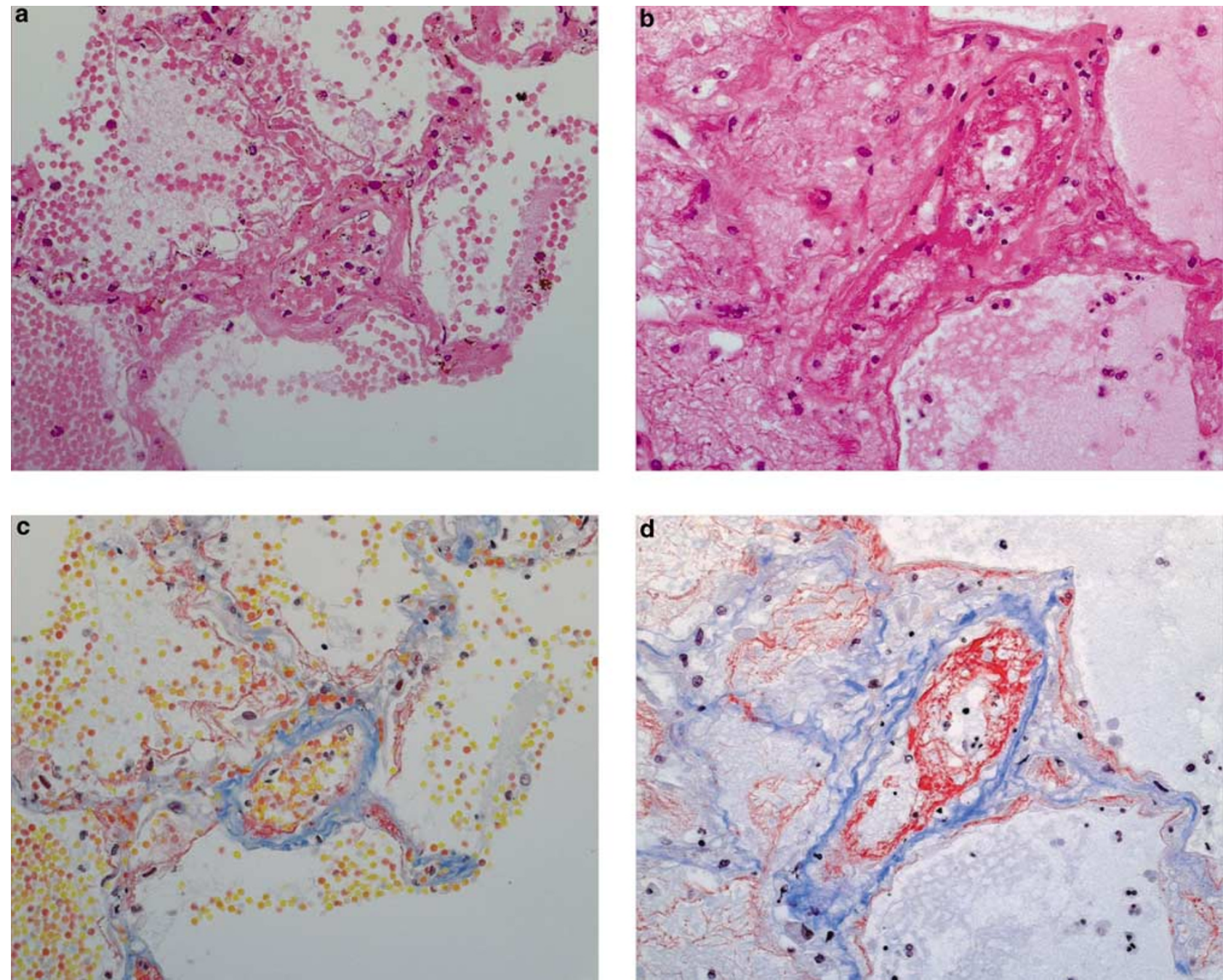

Figure 5 Acute vascular/endothelial injury, with extravasation of fluid and blood cells. (a,b) H\&E, original magnification $\times 400$; (c,d) Martius scarlet blue, original magnification 400x, of the same fields in (a) and (b), respectively, accentuate intravascular and intraalveolar fibrin.

\section{Discussion}

We report here the pulmonary pathology at autopsy of 20 patients who died in the 2003 Toronto SARS outbreak. Of 20 cases in this series, 19 tested positive by RT-PCR for the SARS-CoV in postmortem tissues, confirming that the virus may persist for weeks in patients who ultimately die of the disease, as previously described. ${ }^{16}$ The one patient in whom SARS-CoV was not detected in post-mortem tissues survived more than 3 months from the onset of illness.

In keeping with previous reports, ${ }^{10-15}$ the primary finding in our series of SARS patients was of extensive acute lung injury in varying stages of exudation and organization (Figure 1). This injury was, in general, more prevalent and more extensive among SARS patients than among SARS-CoV-negative patients who died with respiratory symptoms over the same period (Table 1), although there was a significant degree of overlap between the two cohorts. Other associated histologic features, such as pneumocyte hyperplasia, squamous metaplasia, and multinucleated cells, were also present in both groups of patients. Alhough some of these parameters were more prominent in the SARS group as a whole than in the comparison group, this likely reflects differences in duration of illness between the two groups, rather than differences intrinsic to the disease process itself. While not always readily apparent on routine H\&E-stained sections, injury of the pulmonary vasculature was also a prominent feature of most SARS cases (Figures 5 and 6). This was manifested by intravascular fibrin thrombi and thromboemboli, associated in many cases with parenchymal infarcts. Such findings, however, were by no means unique to SARS cases, as similar findings could also be demonstrated in non-SARS cases showing diffuse alveolar damage. Thus, while lethal SARS-CoV infection was virtually always 

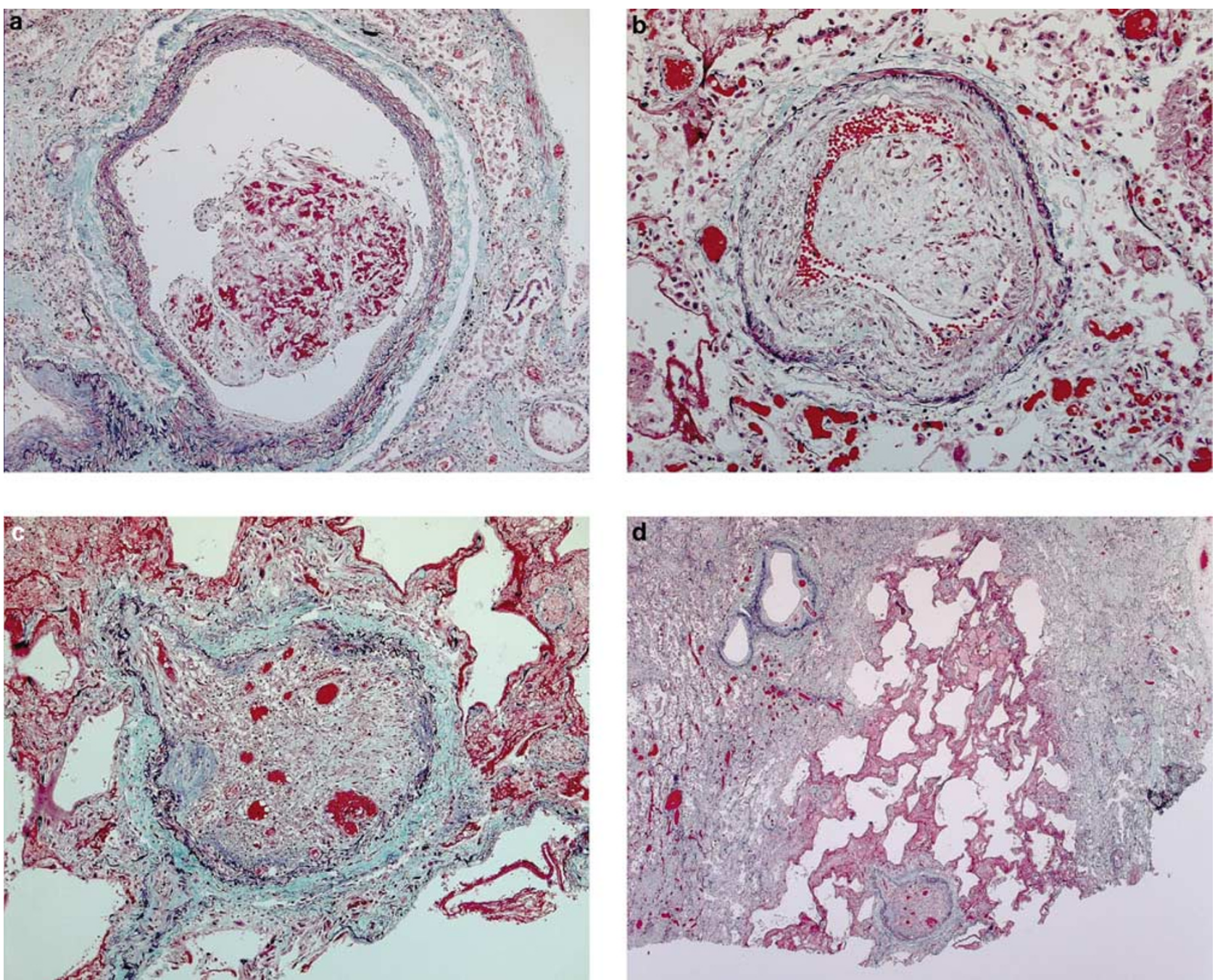

Figure 6 Pulmonary thromboemboli in a SARS patient, duration of illness $=52$ days. (a) Nonocclusive organizing thromboembolus, original magnification $\times 100$; (b) near-occlusive (original magnification $\times 200$ ) and (c) occlusive organized thrombosis with recanalization (original magnification $\times 100$ ); (d) pulmonary infarct associated with occluded vessel (original magnification $\times 25$ ). All sections stained with elastic trichrome.

associated with severe and extensive acute lung injury, we found no specific parameters that would allow definitive diagnosis of SARS-CoV infection prospectively, as opposed to other potential causes of acute respiratory distress syndrome, based on histology alone.

In addition to diffuse alveolar damage, another common feature was the acute fibrinous and organizing pneumonia pattern of lung injury, which was seen in a significant proportion of SARS patients in this series. Described by Beasley and colleagues as a histologic pattern of acute lung injury consisting of intra-alveolar fibrin balls and organizing pneumonia, ${ }^{17}$ this was the predominant pattern of injury in one quarter of our cases, but was not reported in several prior studies describing the pulmonary pathology of SARS. ${ }^{10-14}$ In their recent paper, Tse et al comment briefly on mild bronchiolitis obliterans organizing pneumonia-like changes in the subpleural region of four of seven SARS patients, ${ }^{15}$ but it is unclear from their report whether these show the characteristic acute fibrinous exudates described by Beasley et al, or whether they represent a different process. The reasons for this discrepancy between the present study and prior reports are unclear, but may relate to the longer duration of illness or therapy in patients in the current study compared to previous studies, given that all acute fibrinous and organizing pneumoniapredominant cases in the present series had greater than 20 days' duration of illness (compared to less than 20 days for almost all previously reported cases). The significance of the presence of this pattern of injury, as opposed to classic diffuse alveolar damage alone, is also uncertain. Certainly, the presence of both patterns of injury side-by-side in some cases suggests that the acute fibrinous and organizing pneumonia pattern may simply represent a histologic variant of classic diffuse alveolar damage. However, that some patients exhibited 

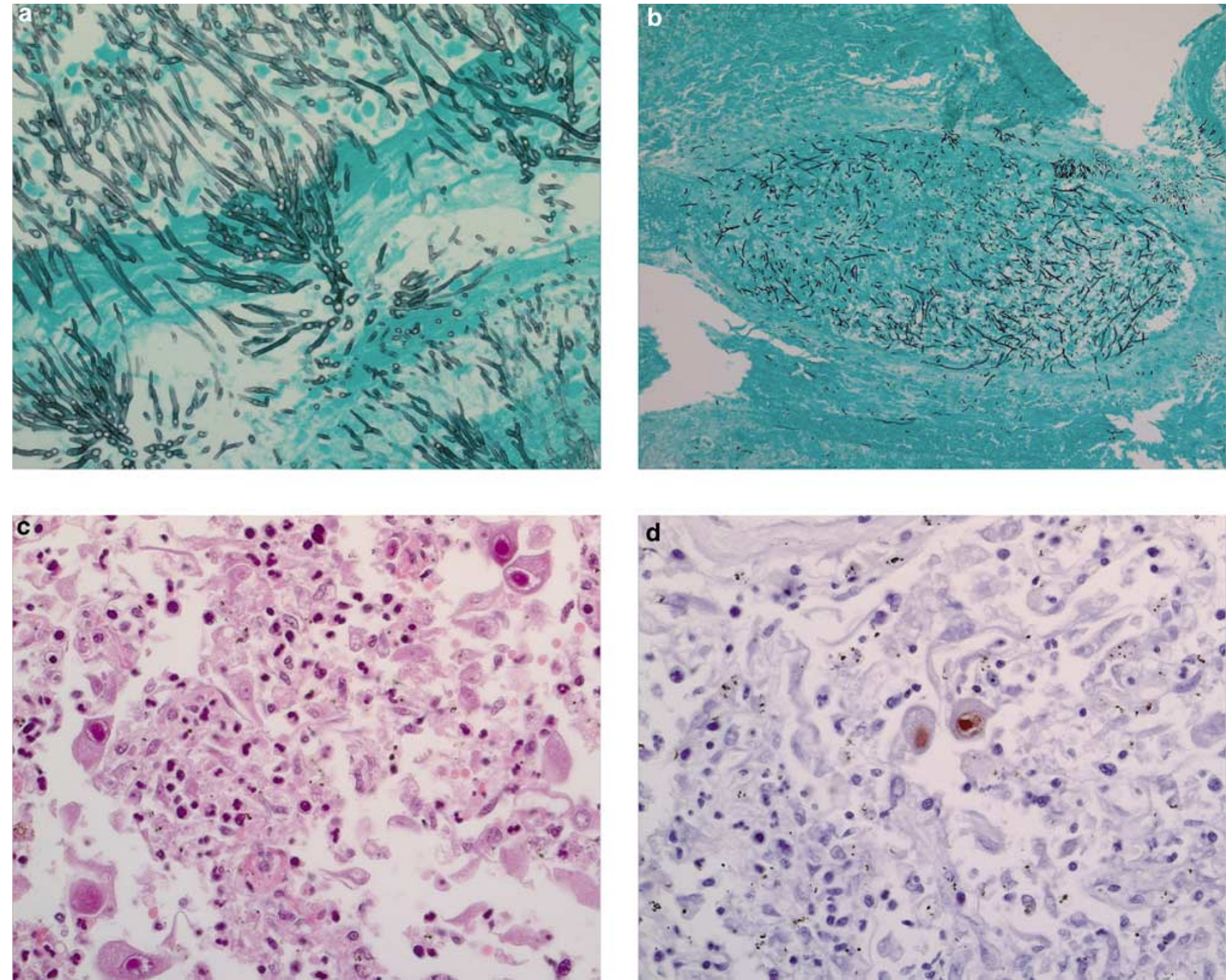

Figure 7 Coinfections in SARS patients. (a) Florid invasive fungal infection consistent with Aspergillosis in a 78-year-old (Gomori methenamine silver, original magnification $\times 400$ ); (b) fungi consistent with Aspergillus spp, with angioinvasion (Gomori methenamine silver, original magnification $\times 100$ ); $(\mathbf{c})$ acute pneumonia, with cells containing intranuclear inclusions consistent with CMV (H\&E, original magnification $\times 400$ ), and staining positive for CMV by immunohistochemistry $(\mathbf{d})$.

one pattern of injury almost exclusively suggests that other factors may be at play, and merits further investigation.

Irrespective of the pattern of injury, patients with longer duration of illness exhibited more fibrosis than those of shorter duration, in keeping with findings from other groups. However, evidence of ongoing acute injury was present in most cases, up to 108 days after the onset of symptoms. Given the persistence of viral RNA in the lungs of all except one patient, such on-going injury could represent the sequelae of persistent SARS-CoV infection, or of injury secondary to other factors such as prolonged mechanical ventilation, or both.

In addition to infection by SARS-CoV, three of 20 patients in this series (15\%) showed confirmed coinfection by other, likely opportunistic, pathogens. Half of the patients in this series also showed patchy acute bronchopneumonia, though causative organisms were not identified. Of the three patients with confirmed coinfection, the lungs of two patients showed widely disseminated invasive fungal infection consistent with Aspergillosis which, in the opinion of the authors, would alone have been sufficient to result in death. While coinfections with bacteria and with fungi such as Aspergillus spp. and Mucor spp. have previously been described in SARS patients, ${ }^{12,14,18}$ histologically identifiable coinfection with another virus has not. As described, one SARS patient in the present series demonstrated the characteristic red-purple intranuclear inclusions of CMV, which subsequently stained positive for CMV by immunohistochemistry (Figure 7c,d). Early reports from China identified virus-like inclusions in presumed SARS patients, but identification by immunohistochemistry was not performed. ${ }^{11,13}$ As similar inclusions have not been demonstrated in any other subsequent studies 
of SARS patients including our current work, these cases may, in retrospect, actually represent coinfection by another virus such as CMV.

In summary, the lungs of fatally infected SARS patients show diffuse alveolar damage and/or acute fibrinous and organizing pneumonia patterns of acute lung injury. Despite the prominence of certain features such as vascular-endothelial injury in SARS lungs, the significant overlap between SARS and non-SARS cases makes it difficult, if not impossible, to differentiate diffuse alveolar damage secondary to SARS-CoV infection vs other etiologies, on the basis of histologic parameters alone. Given the persistence of virus in post-mortem lung tissue in the large majority of cases, ancillary tests such as RT-PCR, immunohistochemistry, or in situ hybridization $^{19}$ should be performed to rule out other etiologies and properly establish the diagnosis in suspected cases. While persistent infection by SARS-CoV may result in on-going lung injury long after the initial onset of symptoms, iatrogenic injury and coexistent superinfections may also contribute significantly to morbidity and mortality in certain patients.

\section{Acknowledgements}

We thank Mr Peter Faure, the pathologists and staff at Toronto Medical Laboratories, University Health Network and the Mount Sinai Hospital, in addition to the Office of the Chief Coroner for Ontario, for their invaluable assistance. Truly, this work would not have been possible without their tireless efforts. We also thank the team that completed the RT-PCR work described in this manuscript, namely Gabriella Farcas and Kevin Kain from the University of Toronto and University Health Network, Tony Mazzulli from the University of Toronto, Toronto Medical Laboratories, and Mount Sinai Hospital, as well as Barbara Willey and Poolak Akhavan from the Toronto Medical Laboratories and Mount Sinai Hospital. This work was funded by Toronto Medical Laboratories, the University Health Network, and in part by a grant from the Canadian Institute of Health Research given to the Canadian SARS Research Network.

\section{Duality of interest}

None declared.

\section{References}

1 Poutanen SM, Low DE, Henry B, et al. Identification of severe acute respiratory syndrome in Canada. N Engl J Med 2003;348:1995-2005.
2 Booth CM, Matukas LM, Tomlinson GA, et al. Clinical features and short-term outcomes of 144 patients with SARS in the greater Toronto area. JAMA 2003;289: 2801-2809.

3 Tang P, Louie M, Richardson SE, et al. Interpretation of diagnostic laboratory tests for severe acute respiratory syndrome: the Toronto experience. Can Med Assoc J 2004;170:47-54.

4 Drosten C, Gunther S, Preiser W, et al. Identification of a novel coronavirus in patients with severe acute respiratory syndrome. $\mathrm{N}$ Engl J Med 2003;348: 1967-1976.

5 Ksiazek TG, Erdman D, Goldsmith CS, et al. A novel coronavirus associated with severe acute respiratory syndrome. N Engl J Med 2003;348:1953-1966.

6 Peiris JS, Lai ST, Poon LL, et al. Coronavirus as a possible cause of severe acute respiratory syndrome. Lancet 2003;361:1319-1325.

7 Kuiken T, Fouchier RA, Schutten M, et al. Newly discovered coronavirus as the primary cause of severe acute respiratory syndrome. Lancet 2003;362: 263-270.

8 Lee N, Hui D, Wu A, et al. A major outbreak of severe acute respiratory syndrome in Hong Kong. N Engl J Med 2003;348:1986-1994.

9 Tsang KW, Ho PL, Ooi GC, et al. A cluster of cases of severe acute respiratory syndrome in Hong Kong. N Engl J Med 2003;348:1977-1985.

10 Nicholls JM, Poon LL, Lee KC, et al. Lung pathology of fatal severe acute respiratory syndrome. Lancet 2003;361:1773-1778.

11 Ding Y, Wang H, Shen H, et al. The clinical pathology of severe acute respiratory syndrome (SARS): a report from China. J Pathol 2003;200:282-289.

12 Franks TJ, Chong PY, Chui P, et al. Lung pathology of severe acute respiratory syndrome (SARS): a study of 8 autopsy cases from Singapore. Hum Pathol 2003;34:743-748.

13 Lang Z, Zhang L, Zhang S, et al. Pathological study on severe acute respiratory syndrome. Chin Med J 2003;116:976-980.

14 Chong PY, Chui P, Ling AE, et al. Analysis of deaths during the Severe Acute Respiratory Syndrome (SARS) epidemic in Singapore. Arch Pathol Lab Med 2004;128:195-204.

15 Tse GM, To KF, Chan PK, et al. Pulmonary pathological features in coronavirus associated severe acute respiratory syndrome (SARS). J Clin Pathol 2004;57: 260-265.

16 Mazzulli T, Farcas GA, Poutanen SM, et al. Severe acute respiratory syndrome-associated coronavirus in lung tissue. Emerging Infect Dis 2004;10:20-24.

17 Beasley MB, Franks TJ, Galvin JR, et al. Acute fibrinous and organizing pneumonia: a histological pattern of lung injury and possible variant of diffuse alveolar damage. Arch Pathol Lab Med 2002;126: 1064-1070.

18 Wang H, Ding Y, Li X, et al. Fatal aspergillosis in a patient with SARS who was treated with corticosteroids. N Engl J Med 2003;349:507-508.

19 To KF, Tong JH, Chan PK, et al. Tissue and cellular tropism of the coronavirus associated with severe acute respiratory syndrome: an in situ hybridization study of fatal cases. J Pathol 2004;202:157-163. 